\title{
OPINIÕES DOS PROFISSIONAIS DE TERAPIA INTENSIVA SOBRE OS PROCEDIMENTOS DE REALIMENTAÇÃO ORAL EM PACIENTES NEUROCRÍTICOS
}

\section{INTENSIVE CARE PROFESSIONALS' OPINIONS ABOUT ORAL INTAKE PROCEDURES IN NEUROCRITICAL PATIENTS}

DOI: $10.5380 /$ rmu.v1i3.40808

Cláudia R. Felicetti-Lordani ${ }^{1}$, Altevir J.G. Tozetto ${ }^{1}$, Silvana T. Duarte ${ }^{2}$, Ana Maria Furkim ${ }^{2}$, Péricles A. D. Duarte ${ }^{3}$

\section{RESUMO}

Procurou-se verificar o procedimento que profissionais e estudantes que atuam em unidade de terapia intensiva (UTI) adotam para realimentação oral em pacientes neurológicos agudos após saída da ventilação mecânica, através de um questionário aplicado a profissionais de saúde (médicos intensivistas, neurologistas e enfermagem da UTI), residentes médicos e estudantes de medicina em um hospital público de ensino. O questionário continha questões sobre critérios de consistência de alimentos e questões para escolher a melhor dieta oral para pacientes com doenças neurológicas agudas. A pesquisa foi respondida por 14 médicos, 9 residentes, 13 estudantes de medicina e 21 profissionais de enfermagem. Foi feita estatística descritiva e comparação entre as médias, com análise univariada. Os parâmetros mais citados para indicar realimentação oral foram: adequado nível de consciência, eficiência da deglutição, e estabilidade gastrointestinal. As consistências de alimentos mais frequentemente citadas para realimentação oral foram pastosos e semipastosos. Contudo, houve várias respostas contraditórias sobre consistência de alimentos, particularmente sobre líquidos. Vários profissionais e estudantes demonstraram pouca preocupação sobre aspectos de disfagia nestes pacientes. Não há concordância sobre procedimentos de realimentação oral no paciente neurocrítico agudo entre profissionais atuantes em terapia intensiva, principalmente sobre selecionar a consistência de alimento a ser testada e no reconhecimento da importância da disfagia neste grupo.

Palavras-chave: Terapia Nutricional; Acidente Vascular Cerebral; Transtornos de Deglutição; Doenças do Sistema Nervoso Central; Traumatismos encefálicos.

\section{ABSTRACT}

Aiming to verify the procedure that intensive care professionals and students adopt for oral intake in acute neurologic patients after mechanical ventilation, a questionnaire was applied to health care professionals (Intensive Care medical and nursing staff, and neurologists), medical residents and medical students in a public teaching hospital. The survey contained questions about food texture criteria, and questions to choosing the best oral diet for patients with neurologic acute diseases. The survey was answered by 14 doctors, 9 residents, 13 medical students and 21 nursing professionals. It was made descriptive statistics, with univariated analysis. The most cited parameters to indicate oral intake were: proper consciousness level, effectiveness of swallowing, and gastrointestinal stability. Food textures most frequently elected to oral intake were soft and semisoft. However, there were several contradictory answers about food texture, mainly about fluids. There is no agreement about oral intake procedures in the acute neurocritical patient among intensive care professionals, mainly about selecting food texture to be tested.

Key Words: Nutrition Therapy; Stroke; Deglutition Disorders; Central Nervous System Diseases; Brain Injuries.

\author{
(1) Nutricionista - (2) Fonoaudióloga - (3) Médico intensivista \\ Contato do Autor / Mail to: \\ Péricles A. D. Duarte - pericles.duarte@unioeste.br \\ Hospital Universitário do Oeste do Paraná - UNIOESTE - Cascavel/PR - STD, CRF, PADD \\ Hospital São Lucas - FAG - Cascavel/PR - AT \\ Curso de Fonoaudiologia - UFSC - Florianópolis/SC - AMF
}




\section{INTRODUÇÃO}

O Suporte Nutricional é crucial no manejo de pacientes criticamente enfermos, e tem sido cada vez mais destacado nos últimos anos, com impacto na morbidade e mortalidade ${ }^{1,2}$. No entanto, o conhecimento e a atenção à nutrição oral e enteral de pacientes na Unidade de Terapia Intensiva (UTI) não são uniformes ${ }^{3}$.

Diversos fatores contribuem para a dificuldade de realimentação oral em pacientes neurológicos agudos: o Traumatismo Crânio-Encefálico (TCE) pode ser associado com o trauma de estruturas faciais e cervicais; drogas (tais como sedativos, relaxantes musculares e corticosteróides) podem comprometer os músculos da deglutição; redução do apetite e do paladar por mecanismos neuroendócrinos; e os efeitos do envelhecimento, particularmente nos pacientes com acidente vascular cerebral (AVC) ${ }^{4-6}$.

Neste contexto, a transição da nutrição enteral ou parenteral para ingestão oral torna-se importante, devido à dificuldade encontrada em alguns grupos de pacientes, principalmente aqueles com ventilação mecânica (VM) prolongada, traqueostomia e condições neurológicas agudas. A principal razão para esta dificuldade é o comprometimento da deglutição. A disfagia orofaríngea pode ser causada por agressão funcional e anatômica de intubação traqueal / traqueostomia ${ }^{7-9}$ ou por lesão neurológica, resultando em incoordenação neuromuscular (disfagia orofaríngea neurogênica) ${ }^{10}$.

A disfagia orofaríngea é geralmente silenciosa, provocando microaspiração sem ser facilmente detectada pela avaliação clínica; entretanto, pode ter conseqüências desastrosas, levando a pneumonia e considerável morbimortalidade ${ }^{11-14}$. Além disso, distúrbios da deglutição podem atrasar a recuperação dos pacientes, devido a causar e agravar a má nutrição e a desidratação ${ }^{15}$.

A Nutrição oral em pacientes com disfagia orofaríngea neurogênica é uma tarefa desafiadora; contudo, nos últimos anos houve progressivo aumento do conhecimento sobre essa condição. Este conhecimento foi gerado por estudos de avaliação da deglutição estado clínico e por métodos invasivos, como a videofluoroscopia da deglutição e nasofibroscopia. Estes métodos têm auxiliado os profissionais de saúde a desenvolver estratégias de posicionamento, reabilitação, sensibilização e escolha de consistências de certos alimentos que podem promover a recuperação funcional do paciente disfágico ${ }^{16}$.

O objetivo deste estudo foi verificar através de um questionário os procedimentos e as opiniões de profissionais de saúde e por estudantes de um hospital universitário sobre as consistências de alimentos a serem oferecidos a pacientes neurológicos agudos na realimentação oral após ventilação mecânica.

\section{MÉTODOS}

Um questionário foi aplicado aos profissionais de saúde e estudantes de medicina (internato) na UTI geral do Hospital Universitário do Oeste do Paraná, em Cascavel/PR. Trata-se de um hospital universitário de 110 leitos; a UTI geral de adultos tinha à época do estudo 9 leitos para pacientes clínicos, cirúrgicos e de trauma. A pesquisa foi realizada durante um mês. Os entrevistadores eram uma nutricionista (CRF) e um estudante de nutrição (AT). A fonoaudióloga (STD) não participou como entrevistadora, porque isso poderia induzir respostas relacionadas à disfagia e deglutição. 0 questionário foi entregue pelo investigador, e o respondedor tinha cerca de 5 a 10 minutos para preenchê-lo.

O exame consistiu de 02 seções. Na primeira parte, havia uma pergunta: "Em sua opinião, quais são os requisitos para se reiniciar a dieta oral nos pacientes neurológicos (encefálicos) hospitalizados que estejam usando nutrição enteral (ou seja, transição de enteral para oral)?". O respondente poderia listar até 03 respostas (descritivas) a esta pergunta. Não havia nenhuma alternativa, ou seja, a pergunta era "aberta".

A segunda parte foi o seguinte sucinto caso clínico: "Paciente com TCE há dez dias; há 3 dias foi extubado (retirado da ventilação mecânica invasiva), tem escala de coma de Glasgow (GCS) de 13 e está atualmente em uso de nutrição enteral." Foram feitas duas perguntas: 1. "Quais os alimentos (nomes) que você indica para iniciar (teste) a dieta oral nesta situação?" 2. "E quais alimentos são contra-indicados?". Também nesta seção (caso clínico), o entrevistado foi solicitado a responder até 03 opções para cada questão. Nenhuma questão tinha alternativas para marcar, ou seja, as perguntas também foram "abertas". Assim, quando foram tabuladas as respostas destas perguntas, foi totalizado mais de $100 \%$, uma vez que poderia existir mais de um tipo de alimento do mesmo grupo de consistência.

As respostas dadas pelos respondentes à segunda parte (caso clínico) eram nomes dos alimentos (carne, mingau de aveia, massas, etc.). Foi usada para subseqüentemente tabular estas respostas uma classificação para a consistência dos alimentos usados pelos serviços de terapia nutricional e de fonoaudiologia do Hospital Universitário (tabela 1). Todos os alimentos mencionados foram classificados a posteriori em uma de quatro categorias pelos investigadores.

A amostra de entrevistados consistiu de médicos (neurologistas, neurocirurgiões, e equipe médica da UTI), estudantes de medicina (internos), residentes da medicina interna e de cirurgia (primeiro e 
segundo ano), e equipe de enfermagem da UTI (enfermeiros e técnicos de enfermagem). Os estudantes estavam no período de treinamento na UTI (estágio) quando a entrevista foi aplicada. Um total de 57 pessoas foram entrevistadas, distribuídas como: 14 médicos, 09 residentes, 13 estudantes, 06 enfermeiras e 15 técnicos de enfermagem.

\section{TABELA 1}

Classificação dos alimentos:

Líquido: Água, chá, sucos, gelatina, etc

Líquido-pastoso (semi-líquido): logurte, sopa, milkshake, etc

Pastoso: Frutas amassadas, purê, mingau, etc

Geral: Carnes, massas, frutas cruas, etc.

Fonte: Hospital Universitário - Depto de Fonoaudiologia e Depto de Nutrição Clínica

Os resultados foram tabulados em planilhas e analisados com o cálculo estatístico de tabelas $2 \times 2$, com comparação de porcentagens. Foi aceito como significante um $p<0,05$. Também foi feita a estatística descritiva, uma vez que alguns dados não permitem comparações precisas.

Os participantes preencheram um consentimento livre e informado. $O$ estudo foi aprovado pelo Comitê de Ética em Pesquisa da Universidade Estadual do Oeste do Paraná para pesquisas que envolvem seres humanos.

\section{RESULTADOS}

Todos os participantes responderam ao questionário. Um médico (do staff médico da UTI) respondeu parcialmente.

Primeira Parte - Requisitos para reintroduzir Dieta Oral a pacientes neurológicos agudos pós VM.

Todos os entrevistados $(n=57)$ responderam a esta pergunta. As respostas continham tanto as " indicações" (por exemplo "nível adequado de consciência") como " ausência de contra-indicações" (por exemplo " ausência de coma"). Houve 39 tipos diferentes de respostas sobre os requisitos para a ingestão oral. Eles foram agrupados em 8 grupos: 1) Nível de consciência; 2) Capacidade de deglutição / tosse; 3) Estabilidade respiratória; 4) Estabilidade gastrointestinal; 5) Estabilidade clínica; 6) Questões relacionadas à traqueostomia ou tempo pós-extubação; 7) Avaliação do nutricionista; 8) Avaliação da fonoaudióloga; 9) Outros.
As respostas mais comuns foram "nível adequado de consciência", relatado 42 vezes, "ter capacidade de engolir", com 17 nomeações, "padrão respiratório satisfatório" (08 vezes) e "tempo de extubação há mais de 24 horas" (07 respostas) .

Quando separados em grupos, os mais citados foram o grupo 1 (Nível de consciência) - 89,5; grupo 2 (Capacidade de deglutição / tosse) - 63,2\%; grupo 3 (Estabilidade respiratória) - 26,3\%, e grupo 4 (Estabilidade gastrointestinal) - 21,1\% (Figura 1). A soma das respostas ultrapassa $100 \%$ porque cada entrevistado podia dar 1 a 3 respostas a esta pergunta, e porque às vezes as respostas foram do mesmo grupo (por exemplo, "adequado nível de consciência" e "Escala de Glasgow acima de 11").

Embora sem significância estatística, houve considerável variabilidade de respostas entre os grupos de profissionais.

Médicos tenderam a enfatizar mais o nível de consciência, a capacidade de deglutir/tosse a estabilidade gastrointestinal, e por outro lado não valorizaram a necessidade de estabilidade respiratória. Os profissionais de enfermagem enfatizaram fortemente a estabilidade respiratória e pouco citaram a estabilidade gastrointestinal. A avaliação fonoaudiológica, por sua vez, só foi mais citada por residentes e acadêmicos de medicina (Figura S-1, material suplementar).

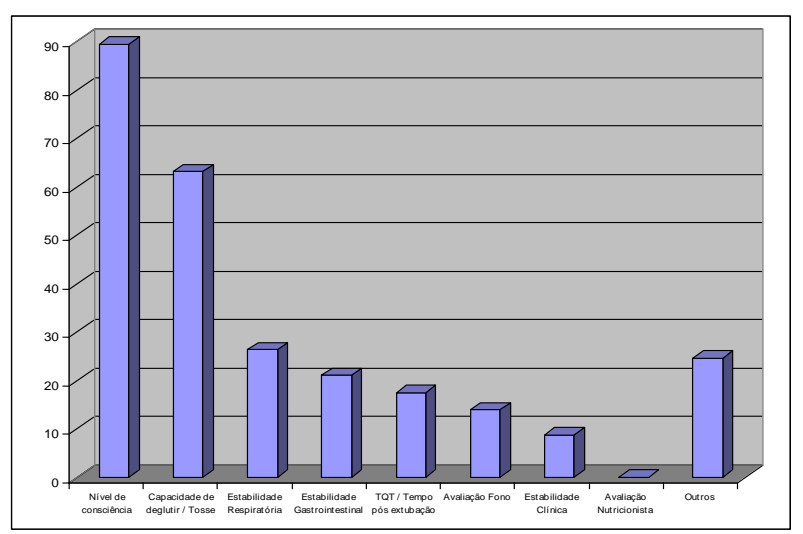

Figura 1.

Requisitos para reiniciar dieta oral. A soma pode exceder $100 \%$

Segunda Parte - Alimentos indicados e contraindicados para a ingesta oral no paciente neurológico agudo.

Também nesta questão, todos $(n=57)$ os entrevistados responderam.

Houve 33 nomes de alimentos relatados como adequados para o cenário clínico (reinício da dieta oral de um paciente neurológico agudo pós-extubação). Os nomes dos alimentos foram posteriormente classificados pelos pesquisadores em 4 grupos (Tabela 1), embora os entrevistados não tinham conhecimento 
desta classificação. Os mais citados como os alimentos indicados foram: "gelatina" (19 respostas), "puré", "sopa" e "suco" (todos com 12 respostas cada), "mingau" (8) e "água" (6 respostas).

Houve um número muito maior de alimentos contra-indicados em comparação com os alimentos indicados - ou seja, a maioria dos entrevistados citou 3 alimentos contra-indicados, mas apenas 1 ou 2 indicados.

Os alimentos contra-indicados mais citados foram: "Carne" (29 respostas), "Arroz" (13), "Feijão" (10), "líquidos" (9 citações), "Água" (6 citações) - Figura S-2 (Material suplementar). Novamente, não houve diferenças estatisticamente significantes entre os grupos de profissionais estudados.

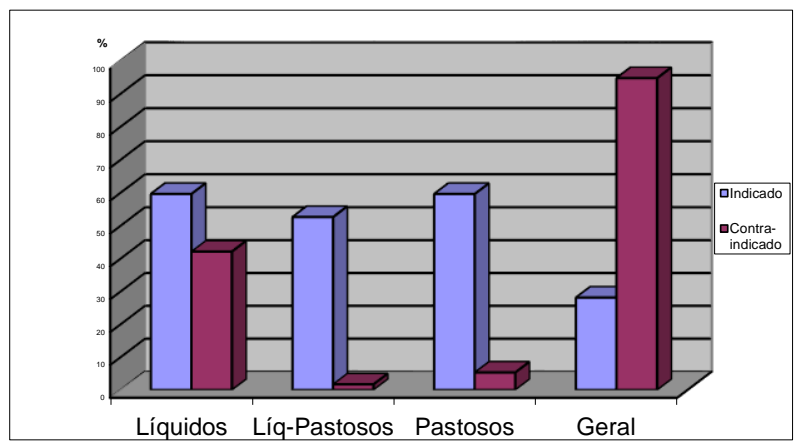

Figura 2.

Alimentos indicados e contra-indicados para reiniciar a dieta oral (de acordo com o grupo). A soma entre os grupos pode exceder $100 \%$.

A Figura 2 mostra os alimentos indicados e contra-indicados de acordo com os grupos de consistência. Entre os 4 grupos de alimento, apenas aqueles classificados como "gerais" tiveram poucas indicações. Ao contrário, os menos citados como contraindicados foram os pastosos e líquido-pastosos (semilíquidos).

Líquidos tiveram uma situação ambígua: foram frequentemente citados como "indicados" e "contraindicados". Quando separados pelos grupos de profissionais (Figura 3), médicos, estudantes e técnicos de enfermagem citaram os líquidos como "indicados", enquanto os residentes e enfermeiros contra-indicaram sua utilização nesse cenário clínico, embora também sem diferenças estatisticamente significantes.

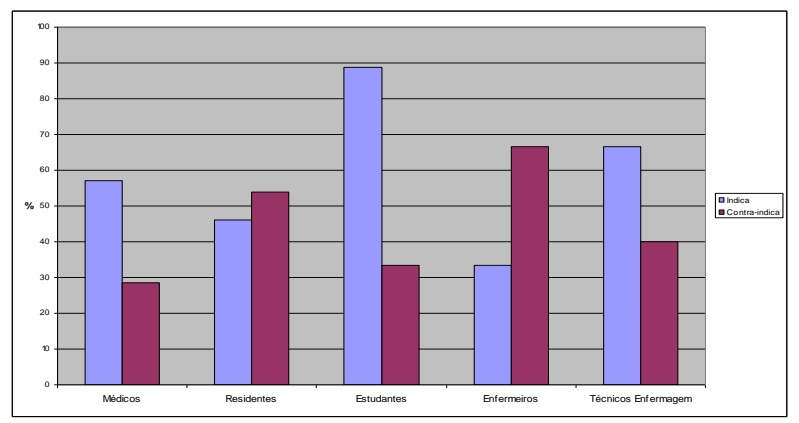

Figura 3.

Líquidos: Indicados e contra-indicados (de acordo com o grupo de profissionais de saúde).

A soma entre os grupos pode exceder $100 \%$.

\section{DISCUSSÃO}

Este estudo procurou verificar o procedimento utilizado por profissionais e estudantes da UTI em relação aos critérios de ingestão oral em pacientes propensos a ter disfagia orofaríngea neurogênica. Ele também procurou avaliar a identificação (por estes profissionais) da consistência dos alimentos frequentemente oferecidos aos pacientes.

Como esperado, o critério mais utilizado pelos entrevistados para permitir o reinício da dieta oral nestes pacientes foi o nível de consciência (Figura 1); não houve diferenças entre os grupos. A maioria dos entrevistados não mencionou quaisquer índices específicos; o mais utilizado foi a escala de consciência de Glasgow. Verificou-se que 3,6\% responderam "Pontuação do Glasgow acima de 10". Baixo nível de consciência é um forte indicador da disfagia e suas complicações $^{12}$, mas não há certeza sobre um valor de escala de Glasgow para dar segurança ao reinício da ingestão oral. De qualquer forma, um paciente com escala de Glasgow de 11 (ou seja, torpor) sugere um nível de consciência ainda pouco provável para fornecer segurança para início da alimentação oral.

A habilidade de deglutir (ou a ausência de disfagia) foi indicada por $63.2 \%$. Embora a literatura seja clara sobre 0 risco da aspiração silenciosa ${ }^{17}$, aproximadamente $1 / 3$ dos entrevistados não considerou a competência da deglutição como chave para reiniciar a alimentação oral neste grupo. Quando comparamos o grupo de médicos/ residentes/ estudantes com o grupo de enfermeiros/ técnicos de enfermagem, há uma diferença significante entre os grupos; sendo uma menor preocupação com disfagia pelo último $(80,5 \% \times 33,3 \%, p=0,001)$.

A presença ou não de traqueostomia e o período de tempo após a retirada da ventilação mecânica são fatores essenciais para a decisão da retomada da alimentação via oral. A ventilação mecânica invasiva e/ ou traqueostomia são importantes fatores de risco para a disfagia orofaríngea, trazendo em conseqüência um risco de até $50 \%$ de aspiração traqueal ${ }^{8}$. Os mecanismos incluem a dessensibilização e edema da laringe; diminuição do reflexo de tosse; atraso no desencadeamento do reflexo de deglutição; fixação traqueo-cutânea; redução da pressurização da laringe durante a deglutição; ferimentos/ ulceração das cordas vocais e laringe; compressão do esôfago pelo "cuff" da cânula; e atrofia dos músculos da deglutição $7,9,13,14,18$ 20 . 
No entanto, estes critérios (intubação traqueal, cuidados com a traqueostomia, e período de tempo após a liberação da VM) foram citados por apenas 17,5\% dos inquiridos. É notável que nenhum residente citou qualquer um desses itens importantes.

Houve grande preocupação entre médicos, residentes e estudantes sobre "a estabilidade gastrointestinal" quando comparada com a equipe de enfermagem $(30,5 \%$ vs $4,8 \%, p=0,050)$. Isto aparentemente reflete uma diferença de ênfase em reiniciar a ingestão oral: em pacientes em pósoperatório de cirurgia abdominal com um nível adequado de consciência e de pequena duração do tempo de ventilação mecânica, há uma tendência para retomar a alimentação por via oral o mais cedo possível21-23, a principal preocupação neste grupo é geralmente com a viabilidade de suturas cirúrgicas e, portanto, para a alimentação por via oral são escolhidos inicialmente alimentos líquidos ${ }^{24}$. No entanto, este não era o caso do grupo de pacientes neste estudo.

A necessidade de avaliação pela fonoaudióloga foi citada por $14,0 \%$ (no grupo de enfermagem foi apenas 4,8\%). Entre os residentes essa taxa foi de 38,5\% ( $p=0,015$ versus todos os grupos). Não houve nenhuma citação sobre a avaliação pelo nutricionista. Esta revelação torna evidente a dificuldade de estruturar a equipe multiprofissional, trabalhando em conjunto no ambiente de UTI. Trabalhar pela cooperação em uma aproximação interdisciplinar pode fornecer um melhor diagnóstico, prevenção e manejo da disfagia e as suas complicações neste grupo dos pacientes ${ }^{16,25}$.

Ao analisar os tipos de alimentos escolhidos para reiniciar a ingestão oral, apenas a dieta geral teve poucas indicações (Figura 2). Não houve diferença estatisticamente significante entre os grupos, embora uma maior percentagem de alunos optou por líquidos, enquanto os médicos tenderam a menor indicação de dieta geral.

Os líquidos foram citados por mais da metade dos entrevistados como um alimento para ser usado para reinício da dieta oral. Ao considerar o nome do alimento, o mais indicado foi gelatina (33,3\%). Embora este estudo não possa discriminar qual foi a definição que cada entrevistado usou para a consistência do alimento escolhido, é sabido que a maioria dos profissionais de saúde e estudantes consideram gelatina como semi-líquido ou pastoso. Entretanto, este estudo refere-se a pacientes neurológicos criticamente enfermos com alta incidência de disfagia neurogênica. Neste contexto, a consistência da gelatina pode mudar dentro da cavidade oral, devido ao longo tempo para engolir. Assim, na prática, a despeito de alguma controvérsia, é habitual se considerar a gelatina como um alimento "líquido" para estes pacientes com maior risco de disfagia, pelo menos nos testes iniciais de reiniciar a ingestão oral ${ }^{26,27}$.
Pacientes com doença neurológica aguda, ventilação mecânica e uso freqüente de traqueostomia apresentam alta incidência de disfagia e aspiração silenciosa, e exigem uma maior atenção e preocupação com a velocidade de reinício da ingestão oral. Além disso, a escolha da dieta repousa sobre alimentos que podem servir como estímulos sensoriais para a mucosa da orofaringe e menor risco de aspiração, como semipastosos e pastosos ${ }^{28-30}$.

Esta contradição (os líquidos são os mais indicados e estão entre as mais contra-indicado) sugere que há alguma dificuldade entre profissionais e estudantes da UTI sobre como reconhecer a consistência dos alimentos para o manejo dos pacientes com alta incidência de disfagia orofaríngea neurogênica.

Quando as respostas dos grupos de profissionais de saúde são discriminadas, é possível constatar que os médicos, estudantes e técnicos de enfermagem indicam líquidos muito frequentemente (muitas vezes na forma de gelatina), ao contrário dos enfermeiros e residentes. Esta abordagem não homogênea indica a necessidade de normatizar os procedimentos sobre a ingestão oral em pacientes internados em UTI.

\section{CONCLUSÕES}

Em um questionário aplicado a profissionais e estudantes na UTI, verificou-se uma grande variabilidade de critérios para indicações e contraindicações de alimentos para reiniciar a ingestão oral de pacientes neurológicos agudos pós-ventilação mecânica, bem como no reconhecimento da consistência do alimento a ser empregado.

Normatização e melhor conhecimento da abordagem e tratamento de pacientes disfágicos em UTI poderia reduzir as complicações do paciente crítico (principalmente pneumonia aspirativa) pela equipe multiprofissional. Enfatiza-se a necessidade da valorização e divulgação do profissional de nutrição no contexto do paciente crítico em UTI.

\section{REFERÊNCIAS BIBLIOGRÁFICAS}

1. Artinian, V; Krayem, H; Digiovine, B. Effects of early enteral feeding on the outcome of critically ill mechanically ventilated patients. Chest 2006; 129: 960-7.

2. Marik P; Zaloga G. Early enteral nutrition in the acutelly ill patients: A systematic review. Crit Care Med 2001; 29: 2264-70.

3. Fulbrook, P; Bongers, A; Albarran, JW. A european survey of enteral nutrition practices and procedures in adult intensive care units. J Clin Nurs 2007; 16: 2132-41.

4. Nematy, M; O’Flynn, Je; Wandrag, L; Brynes, AE; Brett, SJ; Patterson, $\mathrm{M}$; et al. Changes in appetite-related gut hormones in intensive care unit patients: a pilot cohort study. Crit Care 2006; 10: R10-18.

5. Sonies, BC. Oropharyngeal dysphagia in the elderly. Clin Geriatr Med 1992; 8: 569-77. 


\section{ARTIGO ORIGINAL}

6. Brown, CV; Hejl, K; Mandaville, AD; Chaney, PE; Stevenson, G; Smith, $C$. Swallowing dysfunction after mechanical ventilation in trauma patients. J Crit Care 2011; 26 (1):108-13.

7. De Vita, M; Spierer-Rundback, L. Swallowing disorders in patients with prolonged orotracheal intubation or tracheostomy tubes. Crit Care Med 1990; 18 (12): 1328-1330.

8. Romero, CM; Marambio, A; Larrondo, J; Walker, K; Lira, MT; Tobar, E; Cornejo, R; Ruiz, M. Swallowing dysfunction in nonneurologic critically ill patients who require percutaneous dilatational tracheostomy. Chest 2010; 137 (6): 1278-82.

9. Warnecke, T; Suntrup, S; Teismann, IK; Hamacher, C; Oelenberg, S; Dziewas, R. Standardized endoscopic swallowing evaluation for tracheostomy decannulation in critically ill neurologic patients. Crit Care Med 2013; 41 (7): 1728-32.

10. Terré, R; Mearin, F. Prospective evaluation of oro-pharyngeal dysphagia after severe traumatic brain injury. Brain Inj 2007; 21 (13-14): 1411-1417.

11. Goldsmith, T. Evaluation and treatment of swallowing disorders following endotracheal intubation and tracheostomy. Int Anesthesiol Clin 2000; 38 (3): 219-42.

12. Mackay, LE; Morgan, AS; Bernstein, BA. Swallowing disorders in severe brain injury: risk factors affecting return to oral intake. Arch Phys Med Rehabil 1999; 80 (4): 365-71.

13. Macht, $\mathrm{M}$; Wimbish, $\mathrm{T}$; Clark, BJ; Benson, AB; Burnham, EL; Williams, A; et al. Postextubation dysphagia is persistent and associated with poor outcomes in survivors of critical illness. Crit Care 2011; 15 (5): R231.

14. Barquist, E; Brown, M; Cohn, S; Lundy, D; Jackowski, J. Postextubation fiberoptic endoscopic evaluation of swallowing after prolonged endotracheal intubation: a randomized, prospective trial. Crit Care Med 2001; 29 (9): 1710-1713.

15. Clavé, P; Arreola, V; Velasco, M, Quer, M; Castellví, JM; Almirall, $\mathrm{J}$; et al. Diagnosis and treatment of functional oropharyngeal dysphagia. Features of interest to the digestive surgeon. Cir Esp 2007; 82 (2): 62-76.

16. Macht, $\mathrm{M}$; Wimbish, $\mathrm{T}$; Clark, BJ; Benson, AB; Burnham, EL; Williams, $A$; et al. Diagnosis and treatment of post-extubation dysphagia: results from a national survey. J Crit Care 2012; 27 (6): 578-86.

17. Splaingard, M; Hutchins, B; Sulton, L; Chaudhuri, G. Aspiration in rehabilitation patients: videofluoroscopic vs bedside clinical assesment. Arch Phys Med Rehabil 1988; 69 (8): 637-640.
18. McGowan, SL; Gleeson, M; Smith, M; Hirsch, N; Shuldham, CM. A pilot study of fibreoptic endoscopic evaluation of swallowing in patients with cuffed tracheostomies in neurological intensive care. Neurocrit Care 2007; 6 (2): 90-93.

19. Cameron, JL; Reynolds, J; Zuidem, GD. Aspiration in patients with tracheotomies. Surg Gynecol Obstet 1973; 136: 68-70.

20. Tolep, K; Getch, CL; Criner, G. Swallowing dysfunction in patients receiving prolonged mechanical ventilation. Chest 1996; 109 (1): 167-172.

21. Reissman, P; Teoh, TA; Cohen, SM, Weiss, EG; Nogueras, JJ; Wexner, SD . Is early oral feeding safe after elective colorectal surgery? A prospective randomized trial. Ann Surg 1995; 222: 73-77.

22. Lassen, K; Revhaug, A. Early oral nutrition after major upper gastrointestinal surgery: why not? Curr Opin Clin Nutr Metab Care 2006; 9: 613-617.

23. Fukuzawa, J; Terashima, H. Early postoperative oral feeding accelerates upper gastrointestinal anastomotic healing in the rat model. World J Surg 2007; 31: 1234-1239.

24. Petrelli, NJ; Cheng, C; Driscoll, D; Rodriguez-Bigas, MA. Early postoperative oral feeding after colectomy: an analysis of factors that may predict failure. Ann Surg Oncol 2001; 8: 796-800.

25. Odderson, R; McKenna, S. A model for management of patients with stroke during the acute phase. Stroke 1993; 24 (12): 18231827.

26. American Dietetic Association. Manual of clinical dietetics. 6th ed. Chicago: American Dietetic Association, 2000.

27. Dietitians Association of Australia and The Speech Pathology Association of Australia. Texture-modified foods and thickened fluids as used for individuals with dysphagia: Australian standardised labels and definitions. Nutrition \& Dietetics 2007; 64 (Suppl. 2): S53-S76.

28. Bisch, EM; Logemann, JA; Rademaker, AW; Kahrilas, PJ; Lazarus, CL. Pharyngeal effects of bolus volume, viscosity, and temperature in patients with dysphagia resulting from neurologic impairment and in normal subjects. J Speech Hear Res 1994; 37 (5): 1041-1059.

29. Pardoe, EM. Development of a multistage diet for dysphagia. J Am Diet Assoc 1993; 93 (5): 568-571.

30. Peterson, SJ; Tsai, AA; Scala, CM; Sowa, DC; Sheean, PM; Braunschweig, CL. Adequacy of oral intake in critically ill patients 1 week after extubation. J Am Diet Assoc 2010; 110 (3): 427-33. 